\title{
ARTICLE
}

Lymphoma

\section{BET proteolysis targeted chimera-based therapy of novel models of Richter Transformation-diffuse large B-cell lymphoma}

\author{
Warren Fiskus $\mathbb{B}^{1} \cdot$ Christopher P. Mill ${ }^{1}$ - Dimuthu Perera ${ }^{2} \cdot$ Christine Birdwell $^{1} \cdot$ Qing Deng $^{1} \cdot$ Haopeng Yang ${ }^{1}$. \\ Bernardo H. Lara ${ }^{1}$ - Nitin Jain ${ }^{1} \cdot$ Jan Burger $^{1}{ }^{1} \cdot$ Alessandra Ferrajoli $^{1} \cdot$ John A. Davis $^{1} \cdot$ Dyana T. Saenz $^{1}$ • \\ Wendy $\mathrm{Jin}^{1} \cdot$ Cristian Coarfa $\mathbb{D}^{2} \cdot$ Craig M. Crews $\mathbb{D}^{3,4,5} \cdot$ Michael R. Green $\mathbb{D}^{1} \cdot$ Joseph D. Khoury $\mathbb{D}^{1} \cdot$ \\ Kapil N. Bhalla iD ${ }^{1}$
}

Received: 25 November 2020 / Revised: 19 January 2021 / Accepted: 1 February 2021 / Published online: 2 March 2021

(c) The Author(s) 2021. This article is published with open access

\begin{abstract}
Richter Transformation (RT) develops in CLL as an aggressive, therapy-resistant, diffuse large B cell lymphoma (RTDLBCL), commonly clonally-related (CLR) to the concomitant CLL. Lack of available pre-clinical human models has hampered the development of novel therapies for RT-DLBCL. Here, we report the profiles of genetic alterations, chromatin accessibility and active enhancers, gene-expressions and anti-lymphoma drug-sensitivity of three newly established, patientderived, xenograft (PDX) models of RT-DLBCLs, including CLR and clonally-unrelated (CLUR) to concomitant CLL. The CLR and CLUR RT-DLBCL cells display active enhancers, higher single-cell RNA-Seq-determined mRNA, and protein expressions of IRF4, TCF4, and BCL2, as well as increased sensitivity to BET protein inhibitors. CRISPR knockout of IRF4 attenuated c-Myc levels and increased sensitivity to a BET protein inhibitor. Co-treatment with BET inhibitor or BETPROTAC and ibrutinib or venetoclax exerted synergistic in vitro lethality in the RT-DLBCL cells. Finally, as compared to each agent alone, combination therapy with BET-PROTAC and venetoclax significantly reduced lymphoma burden and improved survival of immune-depleted mice engrafted with CLR-RT-DLBCL. These findings highlight a novel, potentially effective therapy for RT-DLBCL.
\end{abstract}

These authors contributed equally: Warren Fiskus, Christopher P. Mill

Supplementary information The online version contains supplementary material available at https://doi.org/10.1038/s41375021-01181-w.

Kapil N. Bhalla

kbhalla@mdanderson.org

1 The University of Texas M.D. Anderson Cancer Center, Houston, TX, USA

2 Department of Molecular and Cellular Biology, Baylor College of Medicine, Houston, TX, USA

3 Department of Molecular, Cellular, and Developmental Biology, Yale University, New Haven, CT, USA

4 Department of Chemistry, Yale University, New Haven, CT, USA

5 Department of Pharmacology, Yale University, New Haven, CT, USA

\section{Introduction}

Richter Transformation (RT) is defined as the development of aggressive DLBCL (mostly ABC-type) in up to $\sim 15 \%$ of patients with antecedent or concurrent diagnosis of chronic lymphocytic leukemia (CLL) [1, 2]. Based on the comparison of immunoglobulin gene rearrangements, $\sim 80 \%$ of RTDLBCLs arise due to a direct clonal evolution of the underlying CLL clone, i.e., clonally related (CLR) RT-DLBCLs, which exhibit poor median survival of 1 year [1-3]. Alternatively, $\sim 20 \%$ of RT-DLBCLs are clonally unrelated (CLUR) to the underlying CLL, arising most likely due to branched clonal evolution from a common pre-CLL progenitor [2-4]. CLUR RT-DLBCLs exhibit a better median survival of 5 years [1-3]. Among the genetic features in CLL that predispose to RT include a stereotypic BCR (subset 8), deletion 17p13, as well as CD38 and LRP4 polymorphisms [5]. There is also an increased risk of RT-DLBCL in CLL with NOTCH1 mutations, bulky lymphadenopathy, and on PET-CT SUV $>5$ in tumor masses, but the risk of developing RT-DLBCL is unrelated to any specific prior therapy of 
CLL [1, 2, 5, 6]. RT-DLBCL is associated with recurrent genetic alterations in TP53 $(\sim 60 \%)$, CDKN2A $(\sim 50 \%)$, NOTCH1 $(\sim 30 \%)$ and MYC $(\sim 40 \%)$, as well as cytogenetic alterations in $17 \mathrm{p}, 9 \mathrm{p} 21$, trisomy 12 , loss of $13 \mathrm{q} 14.3$, 7q31-36.3, 11q22, 14q23.2-q32.33, and near tetraploidy [2, 3, 7-9]. RT-DLBCLs are Epstein-Barr virus (EBV) negative and commonly express high levels of CD20, CD23, PD-1, and PAX5, with low expression of CD5 and CD10 $[2,5,10]$. Clinical studies have documented that chemoimmunotherapy, or treatment with the Bruton's tyrosine kinase (BTK) inhibitor ibrutinib, anti-apoptotic BCL2 inhibitor venetoclax, or with anti-PD1 checkpoint blockade therapy fails to achieve prolonged disease-free survival, and majority of patients relapse with therapy-refractory disease $[2,6,11-15]$. This poor clinical outcome highlights the unmet need to develop and test novel targeted therapies for RTDLBCL. To achieve this, novel in vitro cellular and in vivo patient-derived xenograft (PDX) models of CLR and CLUR RT-DLBCL are essential for elucidating therapeutic targets and for developing and testing novel targeted therapies.

Constitutive activation of B-cell receptor (BCR) signaling and of downstream transcription factors (TFs), especially c-Myc and $\mathrm{NFkB}$, contribute to the growth and survival of lymphoma, including RT-DLBCL cells [2, 3, 16-18]. These TFs bind to their canonical DNA binding sites on enhancers and promoters, recruiting HATs (histone acetyltransferases) to induce acetylation of lysine residues on histone $\mathrm{H} 3$ and $\mathrm{H} 4$ proteins and TFs [19-21]. As a member of the bromodomain extra-terminal (BET) family of reader proteins (BETP), BRD4 binds to acetylated lysine residues on histone proteins and TFs and assembles a complex of co-regulatory proteins [19-21]. These include mediator protein and $\mathrm{P}-\mathrm{TEFb}$ (a heterodimer composed of CDK9 and its regulatory subunit Cyclin T) at super enhancers (SEs)/enhancers (Es) and promoters [19-21]. The kinase activity of CDK9 in P-TEFb phosphorylates serine-2 in the heptad-repeats in the C-terminal domain (CTD) of RNAP2, as well as phosphorylates the negative elongation factors NELF and SPT5, which induces promoter proximal pause-release of RNAP2 to enable productive mRNA transcript elongation [19-23]. Thus, BRD4-CDK9 axis induces RNAP2-mediated transcription of SE/E-driven oncogenes, including MYC, BCL2, Bcl-xL and CDK4/6, which are important for cell growth and survival of RTDLBCL cells [19, 24, 25]. BRD4 also binds acetylated RELA (NFkB-p65) and is essential for NFkB transcriptional activity, necessary for survival of lymphoma cells $[26,27]$. MYC is transcriptionally activated downstream of NOTCH1 mutation, whereas CDKN2A mutation and loss creates a dependency on CDK4 activity through RB phosphorylation [2, 5, 17]. Recently we had also reported that, in ABC-DLBCLs, copy gains of TCF4 gene are common, and BRD4 targeting inhibits TCF4 levels and induces apoptosis in ABC-DLBCL cells [28]. Taken together, these observations suggest that inhibition of BRD4 levels/activity could potentially undermine the transcriptional networks that sustain the growth and survival of RT-DLBCL cells.

In present studies, to address the lack of availability of in vitro cellular and in vivo PDX models of RT-DLBCL, which could be utilized for pre-clinically evaluating the efficacy of novel targeted agents, we successfully established three patient-derived xenograft (PDX) models of RT-DLBCL cells, including clonally related (CLR) (HPRT3) and clonally unrelated (CLUR) (HPRT2) to antecedent CLL. The third RT-DLBCL was a rarer GCB variety of RT-DLBCL (HPRT1). In a previous report, clonal relationships of the RT-DLBCL PDX models to preceding CLL and their sensitivity to therapeutic agents was not characterized [29]. The RT-DLBCL cells for the three PDX models presented here were derived from 3 separate patients with histologicallydocumented RT-DLBCL developing in CLL. The PDX models were established in immune-depleted NSG mice, after tail-vein infusion and engraftment of CD19+ RT-DLBCL cells. Studies presented here also describe the genetic alterations, as well as characterize the epigenomic and transcriptional features of the three PDX models. Additionally, present studies demonstrate marked dependency of CLR, RTDLBCL cells on BRD4-regulated enhancers of oncogenes, including TCF4, IRF4, and MYC. They also demonstrate synergistic lethal activity of BET-proteolysis targeting chimera-based combinations with ibrutinib and venetoclax.

\section{Materials and methods}

\section{Cell lines and cell culture}

Human Richter Transformation DLBCL cells (harvested from the spleen, bone marrow, and liver of PDX-bearing mice) were cultured in RPMI media with $20 \%$ heatinactivated fetal bovine serum (FBS), $1 \%$ penicillin/streptomycin and $1 \%$ non-essential amino acids. Following drug treatments, cells were washed free of the $\operatorname{drug}(\mathrm{s})$ prior to performing the studies described.

\section{Flow cytometry analysis of cell surface markers on RT-DLBCL cells}

To determine the immunophenotype of the RT-DLBCL cells, HPRT3, HPRT2, and HPRT1 cells were harvested from NSG mice. Cells were suspended in $100 \mu \mathrm{L}$ of $0.5 \%$ BSA/PBS and stained with fluorophore-conjugated antiCD19, anti-CD5, anti-CD10, anti-CD20, anti-CD23, antiPD-1 or IgG-isotype controls (BD Biosciences, San Jose, CA). Percent expression of each cell surface marker is reported relative to the respective IgG isotype control. 


\section{RNA isolation and quantitative polymerase chain reaction}

Following the designated treatments with ARV-771 or OTX015, total RNA was isolated from RT-DLBCL cells utilizing a PureLink RNA Mini kit from Ambion, Inc. and reverse transcribed. Quantitative real-time PCR analysis was performed on cDNA using TaqMan probes from Applied Biosystems (Foster City, CA).

\section{Single-cell RNA-Seq analysis of RT-DLBCL cells}

To determine baseline expression of mRNA in the RTDLBCL cells, we performed single cell RNA-Seq analysis utilizing the 10x Genomics Chromium Separator and a Chromium $^{\mathrm{TM}}$ Single Cell $3^{\prime}$ Solution kit followed by nextgeneration sequencing (NGS). Sequencing files were loaded into Cell Ranger and Loupe Cell Browser for clustering, visualization, and analysis.

\section{Statistical analysis}

Significant differences between values obtained in HPRT3, HPRT2, or HPRT1 cells treated with different experimental conditions were determined using the Student's $t$-test in GraphPad V8. For the in vivo mouse models, a two-tailed $t$ test or a Mantel-Cox Rank sum test was utilized for group comparisons. $P$ values of $<0.05$ were assigned significance.

\section{Data sharing statement}

RNA-Seq, ATAC-Seq, and ChIP-Seq datasets have been deposited in GEO as a Super Series under accession \# GSE154463.

Detailed methods for transcriptome analysis, nextgeneration sequencing (NGS) of RT-DLBCL cells by L300 liquid panel, analysis of epigenetic state in RT-DLBCL cells, CRISPR/Cas9-mediated gene editing in RT-DLBCL cells, confocal immunofluorescence microscopy, and RTDLBCL xenograft studies are provided in the Supplemental Methods.

\section{Results}

\section{Generation and biologic features of three PDX models of RT-DLBCLs}

CD19-expressing RT-DLBCL HPRT3, HPRT2, and HPRT1 cells were purified from the core biopsy samples from three patients with histologically-documented RTDLBCL developing in CLL. Prior to establishing their PDX models, we first characterized the biologic features of the
RT-DLBCL cells. Figures $1 \mathrm{~A}-\mathrm{C}$ and S1A, respectively, present the morphologic features, cell cycle phase-distribution, and cell-surface markers of the RT-DLBCL cells. Compared to HPRT3 cells, HPRT2 and HPRT1 cells expressed low CD5, CD23, and PD1, but higher expression of CD20 (Fig. 1C). Flow cytometry and immunohistochemistry analyses revealed that HPRT1 cells exhibited higher \% of cells in the $\mathrm{S}$ and $\mathrm{G} 2 / \mathrm{M}$ phases of the cell cycle and greater expression of Ki-67, TP53, and c-Myc (Figs. 1B, D and S1A). FISH analysis confirmed $5^{\prime} \mathrm{MYC}$ amplification but $3^{\prime}$ MYC deletion in HPRT1 cells (Fig. S1B). HPRT3 and HPRT2 cells were of the most common ABC-DLBCL variety of RT-DLBCL, based on positive MUM/IRF4 and negative CD10 and BCL6 expressions $[1,2]$. In contrast, HPRT1 cells displayed high CD10 and BCL6 expressions, consistent with the rare GCBDLBCL sub-type of RT-DLBCL (Figs. 1C and S1C). In a large cohort of additional 52 RT-DLBCLs managed at MD Anderson Cancer Center, co-expression of CD10 and BCL6 was documented by immunohistochemistry in only one sample, whereas PAX5 and IRF4 were expressed in virtually all RT-DLBCL samples (Tables S1 and S2). All three RT-DLBCL cell-types lacked EB virus DNA or the expression of EBNA2 protein (Fig. S1D, E) [2]. Clonal relationship of each of the three RT-DLBCL samples to their antecedent CLL samples was assessed by analyzing and comparing their immunoglobulin genes to those of the preceding CLL cells. Notably, HPRT3 was clonally-related to its antecedent CLL, whereas HPRT2 was clonallyunrelated (Table S3). Clonal relationship of HPRT1 cells to its antecedent CLL could not be established because of the lack of availability of the DNA from the preceding CLL cells. Next, we established PDX models of luciferasetransduced, CD19+ HPRT3, HPRT2, and HPRT1 cells, following tail-vein infusion and engraftment in immunedepleted NSG mice (Fig. 1E). The RT-DLBCL cells grew in the bone marrow, spleen and liver and caused marked splenomegaly and hepatomegaly, requiring euthanasia of the mice 4 to 6 weeks after engraftment (Fig. 1F).

\section{Cytogenetics, gene-copy number variations, and genetic mutations in the RT-DLBCLs}

Cytogenetic analysis showed large numbers of karyotypic abnormalities in the three DLBCLs, especially pronounced in HPRT3 cells (Fig. S2A). Chromosomes showing tetrasomies were prevalent in HPRT3, whereas trisomies were present in HPRT3 and HPRT2 RT-DLBCLs (Fig. S2A). Array-CGH analysis also demonstrated gains and losses of chromosomal regions, again more pronounced in HPRT3 compared to HPRT2 and HPRT1 cells (Fig. S2B). We also conducted low-pass whole-genome sequencing to determine regions of amplification and losses [28]. All three 

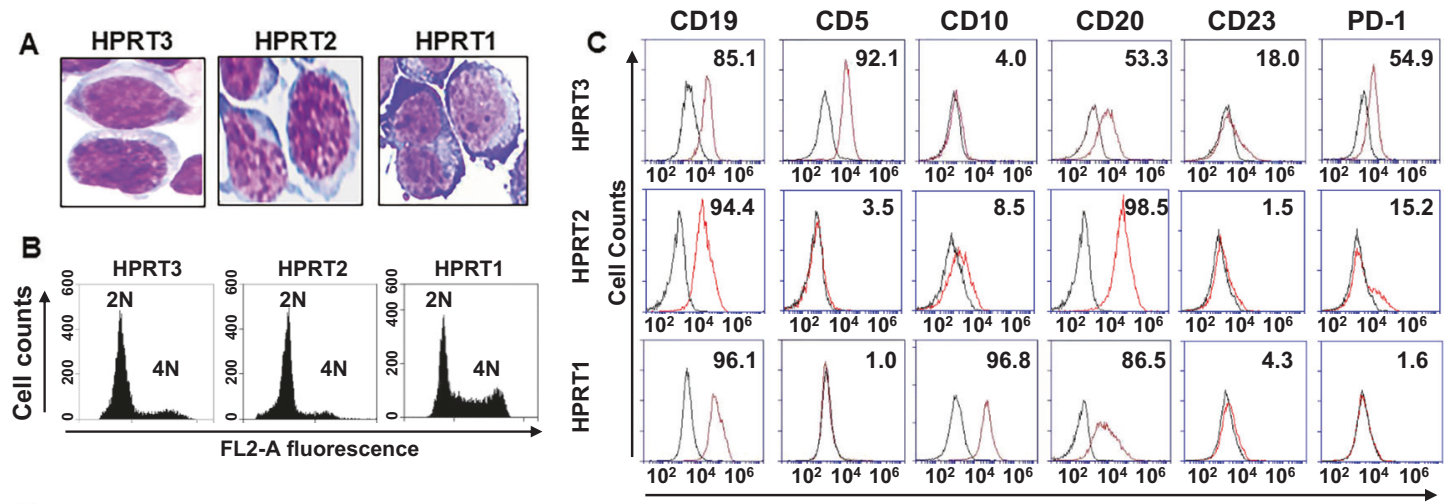

D

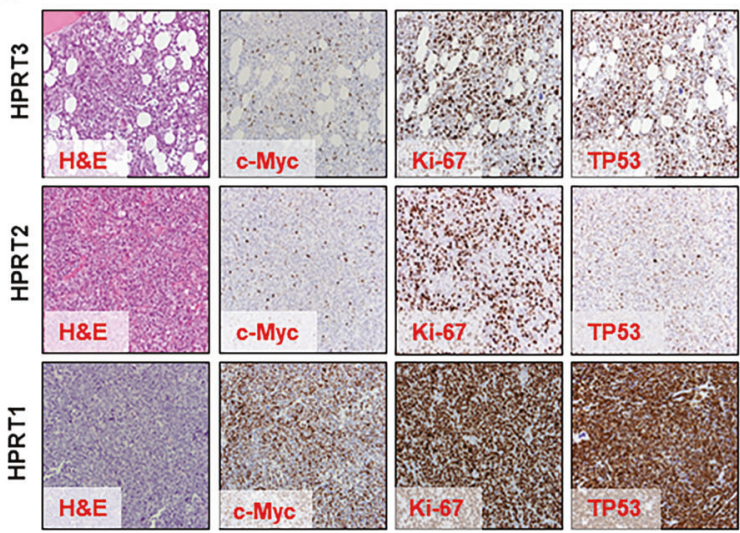

Fluorescence intensity (black line: IgG control)

E
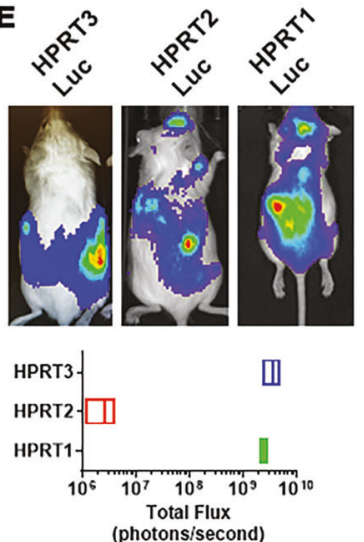
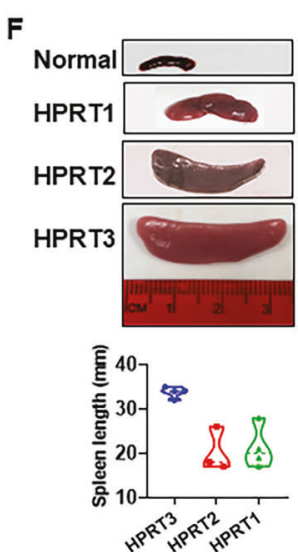

Fig. 1 Phenotypic characterization of human HPRT3, HPRT2, and HPRT1 Richter Transformation PDX models. A RT-DLBCL cells from PDX models were cytospun onto glass slides at $500 \mathrm{rpm}$ utilizing a cyto-centrifuge. Cells were fixed with Fast Green and stained with hematoxylin and eosin. Original magnification is $\times 40$. Cell images were obtained with a CCD camera mounted on a microscope. B Representative cell cycle status histograms of HPRT3, HPRT2, and HPRT1 cells. RT-DLBCL cells were harvested, washed with $1 \times$ PBS and fixed in $70 \%$ molecular grade ethanol overnight at $-20^{\circ} \mathrm{C}$. Following this, RT-DLBCL cells were washed with $1 \times$ PBS and stained with $1 \mathrm{mg} / \mathrm{mL}$ propidium iodide in Triton-PBS buffer. Cells were analyzed by flow cytometry. C RT-DLBCL cells were harvested and stained with the indicated cell surface markers or the corresponding IgG isotype control. The numbers beside the histograms indicate the $\%$ of cells expressing each surface marker relative to the $\mathrm{IgG}$ isotype control. D Immunohistochemical evaluation of c-Myc, KI67, and TP53 expression in RT cells. E HPRT3, HPRT2, and HPRT1 cells were luciferized to enable tracking of their in vivo growth in NSG mice. Representative images of engrafted luciferaseexpressing RT-DLBCL PDX cells 4 weeks after cell implantation are shown. The total flux (photons/sec) from 2 to 3 mice with each PDX is shown. F Representative spleens from HPRT1, HPRT2, and HPRT3-engrafted mice compared to a normal mouse spleen approximately 6 weeks after cell implantation. The range of spleen sizes from 3 mice with each PDX model is shown in the violin plot.

factors, epigenetic regulators, DNA damage/repair enzymes, signaling enzymes, and their regulators.

\section{Epigenomic and gene-expression diversity of the RT-DLBCL cells}

To elucidate the impact of the large array of genetic alterations on chromatin accessibility, enhancer activity, and on gene-expression profiles, we performed ATAC-Seq, ChIP-Seq with H3K27Ac and BRD4 antibodies, and singlecell (sc) RNA-Seq analyses in HPRT3, HPRT2, and HPRT1 cells [28, 30, 31]. Utilizing anti-H3K27Ac ChIPSeq analysis, we evaluated the active chromatin as signaldensity plots of H3K27Ac mark on the chromatin of RTDLBCL cells compared to publicly available H3K27Ac 
A
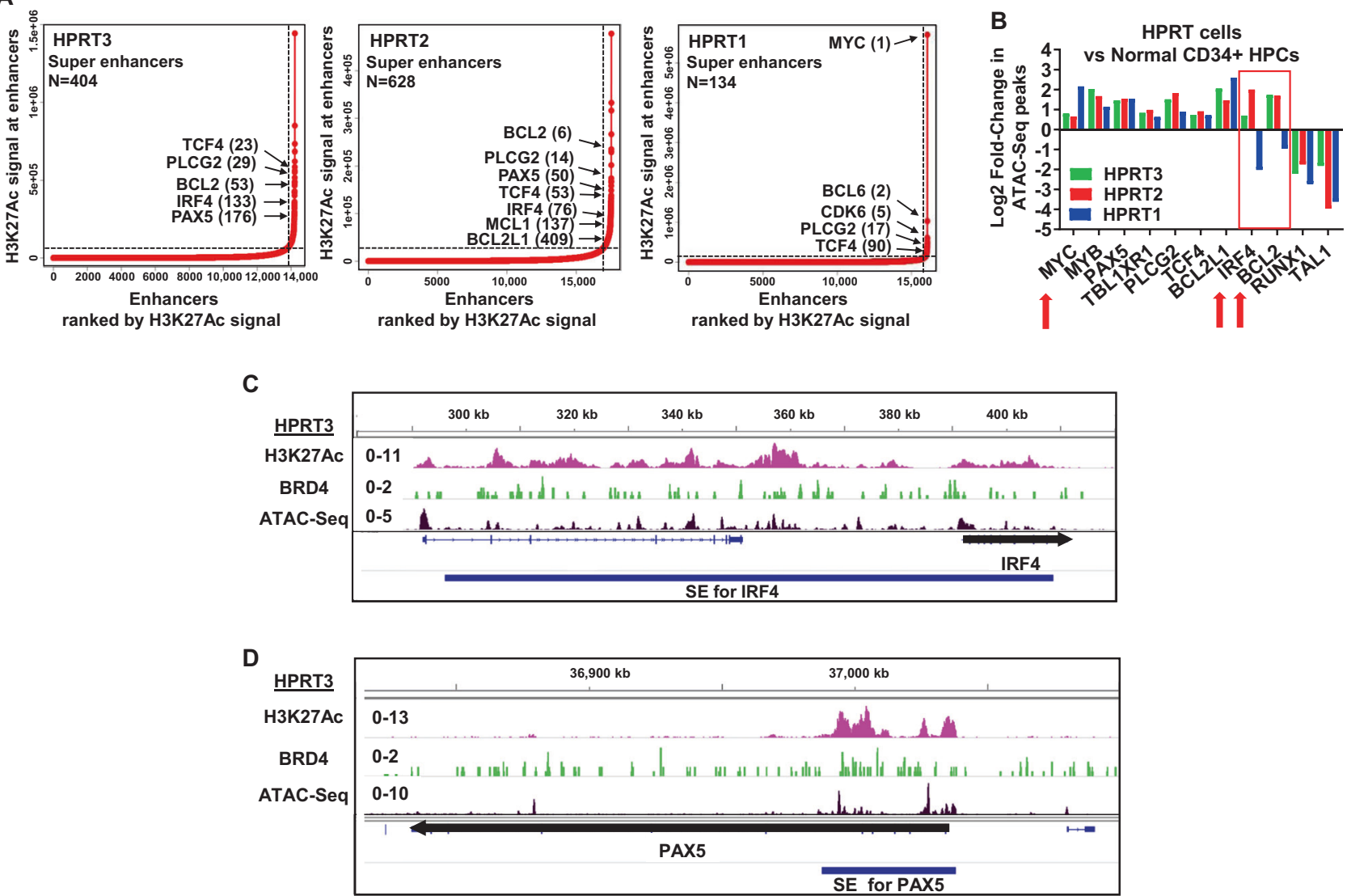

Fig. 2 Epigenome and super-enhancer analysis in HPRT3, HPRT2, and HPRT1 cells and sequence tag density plots of H3K27Ac and BRD4 occupancy and chromatin accessibility at the IRF4 and PAX5 genes in HPRT3-DLBCL cells. A H3K27Ac ChIPSeq analysis was performed on the chromatin from RT-DLBCL cells. Ranked ordering of SE (ROSE) analysis was performed utilizing the $\mathrm{H} 3 \mathrm{~K} 27 \mathrm{Ac}$ sequence-tag density and H3K27Ac signal at enhancers. B ATAC-Seq analysis was performed on the nuclei from RT-DLBCL cells to determine chromatin accessibility. Publicly available ATACSeq data from normal CD34+ HPCs were downloaded from GEO.

ChIP-Seq data from normal CD34+ hematopoietic progenitor cells (HPCs) (GSM772870, GSM772885, and GSM772894). Fig. S3A shows the average, normalized read-density across all SEs/Es as markedly increased sequence-tag densities in HPRT3 > HPRT1 > HPRT2 > normal CD34+ HPCs, compared to the read-densities on either side of the SEs/Es. Taking into account the SE/E score reflecting both enhancer-size and density of reads, we identified several SEs with high scores in HPRT3, HPRT2, and HPRT1 cells, as shown in the 'ROSE plots' in Fig. 2A. The top 25 super enhancers in HPRT3, HPRT2, and HPRT1 are shown in Table S8A-C. Whereas SEs of TCF4 and PLCG2 scored high in all three RT-DLBCL cells, MYC, BCL6, and CDK6 SEs scored high only in HPRT1 cells $[28,32,33]$. HPRT3 and HPRT2 also demonstrated active SEs of BCL2, PAX5, and IRF4 (Fig. 2A) [25, 32-34]. Heat map of the signal density determined by ATAC-Seq
Log2 fold-changes in peaks from selected RT-relevant genes as determined by ATAC-Seq analysis are shown. Red arrows indicate significant differences in accessibility in the three RT-DLBCL subtypes compared to each other and normal CD34+ cells. C, D Integrated Genome Viewer (IGV) plots showing H3K27Ac and BRD4 ChIP-Seq signal density and ATAC-Seq determined chromatin accessibility at the IRF4 and PAX5 genes in clonally-related HPRT3DLBCL cells. The blue bars beneath the signal tracks indicate the position of the super enhancer for IRF4 and PAX5 as determined by ROSE analysis.

analysis showed markedly altered signal intensity in the three RT-DLBCL cell-types, as compared to publiclyavailable ATAC-Seq data from normal CD34+ HPC cells (GSE18927) (Fig. S3B). Loci showing log2 fold-alterations in ATAC-Seq peaks in HPRT3, HPRT2, and HPRT1 cells, versus normal CD34+ HPC ATAC-Seq peaks, are depicted in Fig. 2B. Compared to HPRT3 and HPRT2 cells, chromatin of IRF4 and BCL2 genes was less, whereas that of MYC was more accessible in HPRT1 cells (Fig. 2B). Figures $2 \mathrm{C}, \mathrm{D}$ and $\mathrm{S} 3 \mathrm{C}, \mathrm{D}$ demonstrate ChIP-Seq analysesdetermined signal-density plots of $\mathrm{H} 3 \mathrm{~K} 27 \mathrm{Ac}$ and BRD4, as well as ATAC-Seq-determined peak-density at the MYC and the adjacent PVT1 gene [31], as well as at the SEs for IRF4, TCF4, and PAX5 genes. As shown here, in HPRT3 cells, greater $\mathrm{H} 3 \mathrm{~K} 27 \mathrm{Ac}$ and BRD4 peaks and increased chromatin accessibility was observed on the E of PVT1, as well as on the SEs of TCF4, IRF4, and PAX5 genes. Similar 
findings were also observed in HPRT2 cells (Fig. S3E-H). In HPRT1 cells, in addition to MYC gene amplification, the Es of MYC and PVT1 genes showed a marked increase in H3K27Ac and BRD4 peaks and augmented chromatin accessibility, especially at the Es 1,2, and 3 for the MYC gene (Fig. S3I). Next, we performed single-cell (sc) RNA sequencing, and generated t-SNE plots from the sequence reads based on similar mRNA expression levels in the clustered HPRT3 (12 clusters), HPRT2 (7 clusters) and HPRT1 cells (7 clusters) (Fig. S4A-C). Panels below the tSNE plots show the number of clusters and number of cells per cluster for HPRT3, HPRT2, and HPRT1 cells. Fig. S4D demonstrates selected t-SNE plots of the baseline mRNA expressions of TCF4, MYC, IRF4, PAX5, BCL2, and BCL2L1 across all clusters in HPRT3, HPRT2, and HPRT1 cells. As shown here, whereas TCF4 expression was high in all clusters in all three RT-DLBCLs, MYC mRNA was clearly expressed at high levels in HPRT1 cells. Additional t-SNE plots of baseline mRNA expressions of 16 genes across the clusters in HPRT3, HPRT2, and HPRT1 cells are also presented in Fig. S4E-G. Cells from each PDX were also clustered and displayed using Uniform Manifold Approximation and Projection (UMAP), with clusters shown on Fig. S4H-J and cluster features provided in Table S9. Features of clusters included BRD4-regulated genes such as MYC (HPRT1, HPRT3), MCL1 (HPRT1, HPRT3), BIRC3 (HPRT1, HPRT3), IRF4 (HPRT2, HPRT3), and TCF4 (HPRT2, HPRT3), as shown on UMAP feature plots in Figures S4K-M.

\section{Disparate sensitivity of RT-DLBCL cells to ibrutinib or BH3-mimetics correlates with protein expressions regulating their activity}

Previous reports had highlighted differential activity and response of RT-DLBCL cells to novel targeted agents such as BCL2 inhibitor venetoclax, and BTK inhibitor ibrutinib [11, 12, 14]. As shown in Fig. 3A, unlike the high level of sensitivity of CLL cells documented for venetoclax [35], the three RT-DLBCL cells were relatively less sensitive to venetoclax. Nonetheless, HPRT3 and HPRT2 were relatively more susceptible to venetoclax than HPRT1 cells. This correlated with higher BCL2, BAX, BAK, and BIM levels in HPRT3 and HPRT2 compared to HPRT1 cells (Fig. 3B). Higher Bcl-xL expression also correlated with increased sensitivity of HPRT3 and HPRT2 versus HPRT1 cells to a Bcl-xL-specific inhibitor A-1155463 (Fig. 3C) [36]. Conversely, higher MCL1 levels correlated with greater sensitivity of HPRT1 cells, compared to HPRT3 and HPRT2 cells, to an MCL1-specific inhibitor AZD-5991 (Fig. 3D) [36]. Notably, HPRT3 and HPRT2 were resistant to ibrutinib, as compared to HPRT1 cells
(Fig. 3E). As was shown in a previous report, this is likely due to activation of the alternative MAP3K14-NFkB pathway, instead of the classical BCR-BTK-NFkB activation pathway, in HPRT3 and HPRT2 cells [37]. The alternative pathway involves increased processing of p100 to p52 by MAP3K14 (NIK kinase) resulting in activation of NFkB2 [37], which was noted in HPRT3 and HPRT2 cells (Fig. 3F). Despite expressing elevated levels of p-AKT, HPRT1 cells exhibited increased sensitivity to ibrutinib, which was consistent with increased p-BTK and p-PLC $\gamma 2$ levels, suggesting increased BCR activity [38-40]. Compared to HPRT3 and HPRT2, HPRT1 cells also exhibited higher sensitivity to lenalidomide (Fig. 3G) [41]. This was associated with increased levels of cereblon and IKZF1/3 in HPRT1 cells compared to HPRT3 and HPRT2 cells (Fig. 4C, vide infra) [41, 42]. In contrast, the three RTDLBCL cells displayed disparate level of sensitivity to the topoisomerase II inhibitor doxorubicin (Fig. 3H). Collectively, these findings demonstrated that the three RTDLBCLs are differentially sensitive to several targeted agents, which correlated with expression levels of genes that regulate sensitivity to the targeted agents.

\section{Sensitivity of RT-DLBCL cells to BET inhibitor or its combination with ibrutinib or venetoclax}

We previously reported that BET inhibitors (BETis) either alone or in combinations with ibrutinib or venetoclax induce apoptosis in NHL including DLBCLs [27, 43]. Based on this, we determined the lethal activity of BETi OTX015 or ABBV-075 against the three RT-DLBCL cells. Treatment with OTX015 and ABBV-075 induced more lethality in HPRT1, compared to HPRT3 and HPRT2 cells (Fig. 4A, B). This correlated with higher levels of BRD4, cMyc, and TCF4, but markedly lower levels of IRF4 in HPRT1 compared to HPRT3 and HPRT2 cells (Fig. 4C). Levels of DUB3, SPOP and TRIM33/24, previously reported to be involved in regulating sensitivity to BETis [44-46], were neither significantly different nor altered by OTX015 treatment in the three RT-DLBCL cells (Fig. S5A). RNA-Seq analysis showed that following treatment with OTX015 larger numbers of mRNA levels were up or downregulated in HPRT1 compared to HPRT3 and HPRT2 cells (Fig. S5B, C). The Venn diagram in Fig. S5C shows that 540 genes were commonly depleted whereas 574 genes were commonly induced by OTX015 treatment of the three RT-DLBCL cells. Among the mRNA expressions demonstrating greater than $\log 2$-fold inhibition (e.g., -0.3219) in HPRT1 cells were those of SLC19A1, MYC, MYB, BIRC3, BTK, CDK4/6, NFkB1, IRF4, and BCL2L1, while CDKN1A, PMAIP1, and HEXIM1 mRNA levels showed log2-fold increases (Fig. S5D). QPCR 
A

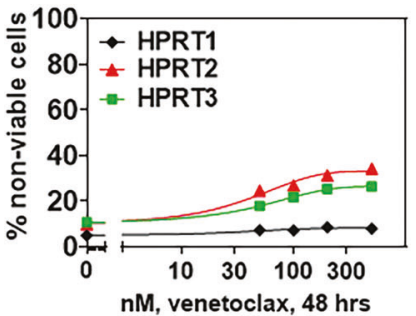

B
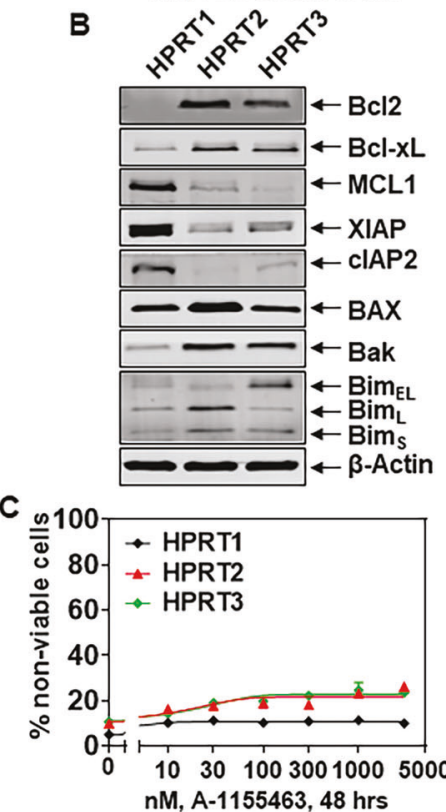

D

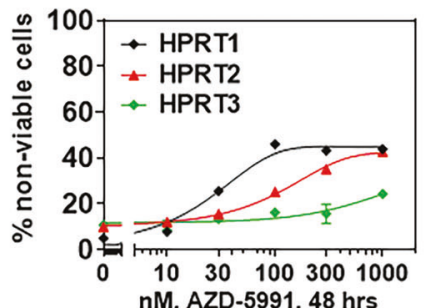

E

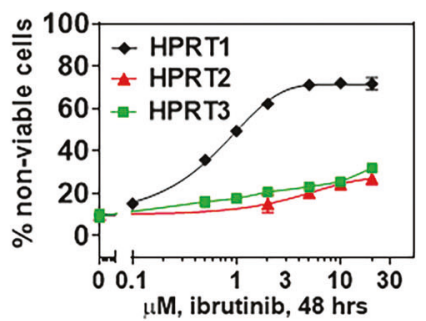

G

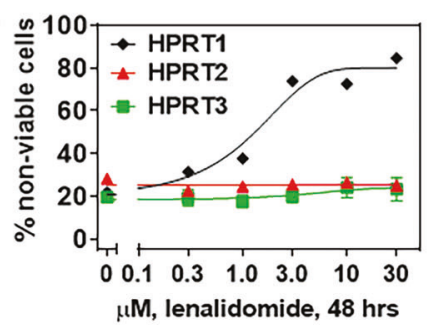

$\mathbf{F}$

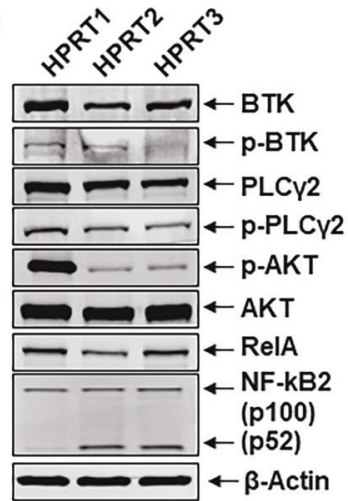

$\mathrm{H}$

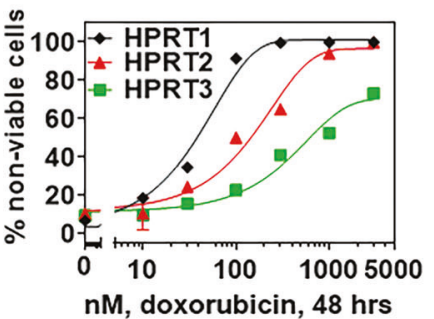

Fig. 3 RT-DLBCL cells exhibit a diverse range of sensitivity to venetoclax, ibrutinib, lenalidomide, and doxorubicin. A RTDLBCL cells were treated with the indicated concentrations of BCL2 inhibitor venetoclax, for $48 \mathrm{~h}$. At the end of treatment, cells were washed with $1 \times$ PBS and stained with To-Pro- 3 iodide. The $\%$ of non-viable cells was determined by flow cytometry. Mean of three experiments \pm SEM. B Expression levels of BCL2 family proteins in HPRT1, HPRT2, and HPRT3 cells. The expression levels of $\beta$-Actin in the lysates served as the loading control. C-E RT-DLBCL cells were treated with the indicated concentrations of Bcl-xL-specific inhibitor A-1155463, MCL1 inhibitor AZD-5991, or ibrutinib, for 48 h. At the end of treatment, cells were washed with $1 \times$ PBS and stained with To-Pro- 3 iodide. The $\%$ of non-viable cells was determined by flow cytometry. Mean of three experiments \pm SEM. F Expression levels of B-cell receptor pathway proteins in HPRT1, HPRT2, and HPRT3 cells. The expression levels of $\beta$-Actin in the lysates served as the loading control. G-H RT-DLBCL cells were treated with the indicated concentrations of lenalidomide or doxorubicin for $48 \mathrm{~h}$. At the end of treatment, cells were washed with $1 \times$ PBS and stained with To-Pro-3 iodide. The $\%$ of non-viable cells was determined by flow cytometry. Mean of three experiments \pm SEM.

sensitivity to OTX015 in IRF4 KO cells, suggesting that IRF4 overexpression attenuates BETi-induced lethality in RT-DLBCL cells (Fig. 4F, G). We also determined the effect of ectopic overexpression of c-Myc, (driven by an EF1 $\alpha$ promoter) via nucleofection into HPRT3 and HPRT2 cells. Although c-Myc overexpression increased \% non-viable cells, it significantly increased OTX015-induced lethality in HPRT3 and HPRT2 cells (Fig. S6A, B). Additionally, we determined the effect of CDK9 inhibitor NVP-2 and AZD4573 on the viability of the RT-DLBCL cells. As shown in Fig. S7A, B, treatment with NVP-2 and AZD4573 dose-dependently induced loss of viability of the RTDLBCL cells. Treatment with NVP-2 also reduced the levels of serine 2 (S2) phosphorylated RNAP2, IRF4, cMyc, MCL1 and Bcl-xL, while increasing the levels of cleaved PARP in HPRT3, HPRT2, and HPRT1 cells depletion of IRF4 and c-Myc significantly increased 
A

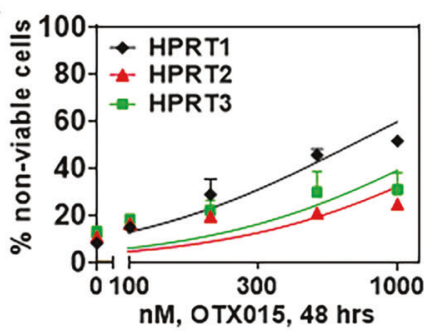

B

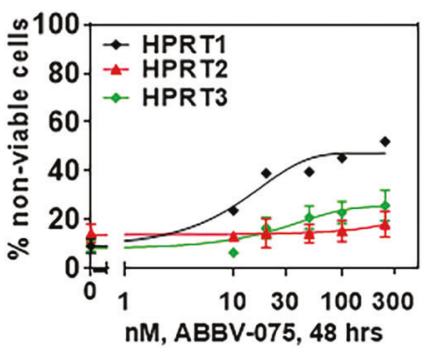

$\mathbf{F}$

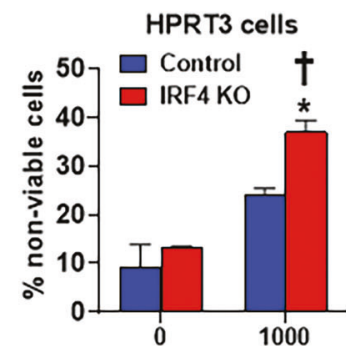

nM, OTX015, 48 hrs

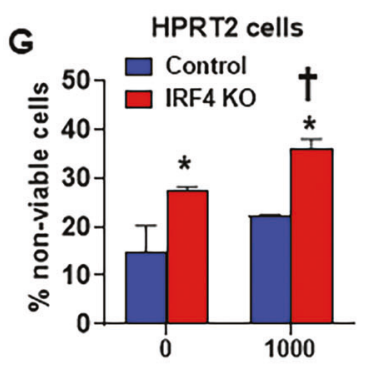

nM, OTX015, 48 hrs

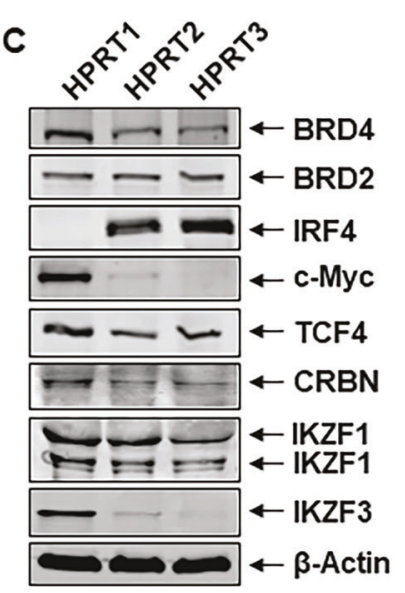

D

HPRT3
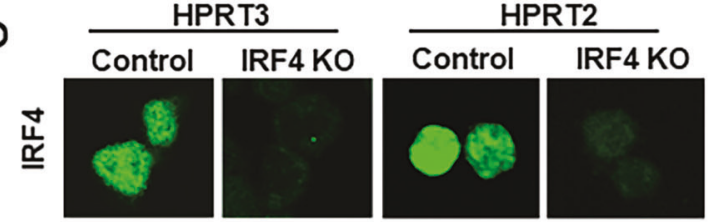

$\sum_{j}^{0}$
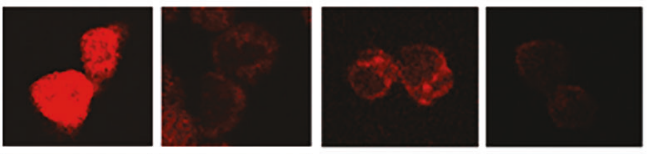

$\frac{\bar{a}}{2}$
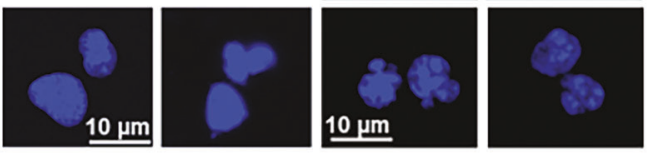

E
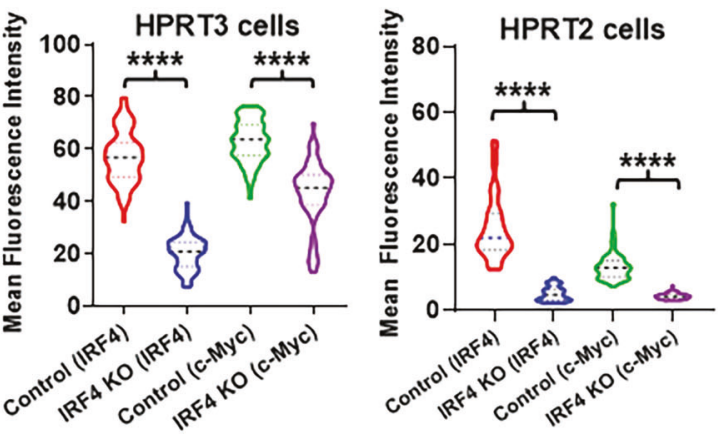

H

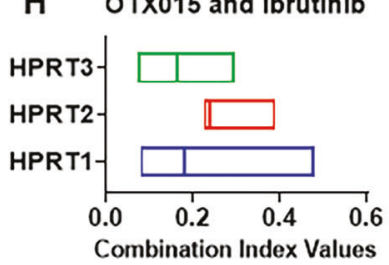

I OTX015 and venetoclaX

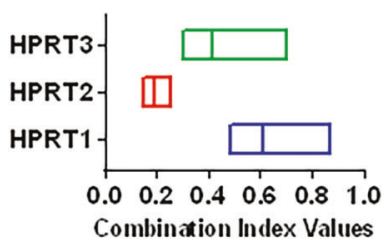

microns. E Mean fluorescence intensity of IRF4 and c-Myc expression in HPRT3 and HPRT2 cells from two independent experiments transfected as in (D) and imaged by confocal microscopy. F-G HPRT3 and HPRT2 cells were transfected as in (D), and incubated on HS5 cells for $72 \mathrm{~h}$. Following this, cells were plated and treated with OTX015 for $48 \mathrm{~h}$. At the end of treatment, cells were washed with $1 \times$ PBS and stained with To-Pro-3 iodide. The $\%$ of To-Pro-3 iodidepositive, non-viable cells was determined by flow cytometry. *Indicates values that are significantly greater in HPRT3 or HPRT2 cells transfected with IRF4 sgRNAs and treated with OTX015 than cells transfected with sgNeg (Control) and treated with OTX015 determined by two-tailed, unpaired $t$-test. ${ }^{\dagger}$ Indicates values significantly greater in OTX015-treated cells transfected with IRF4 sgRNAs (IRF4 KO) than untreated cells determined by two-tailed, unpaired $t$-test. H, I RTDLBCL cells were treated with OTX015 (dose range: $250-1000 \mathrm{nM}$ ) and ibrutinib (dose range: $2-10 \mu \mathrm{M}$ ) or venetoclax (dose range: $20-500 \mathrm{nM}$ ) for $48 \mathrm{~h}$. The $\%$ non-viable cells was determined by flow cytometry. Combination index (CI) values were calculated with CompuSyn and boxplots were generated with GraphPad V8. CI values $<1.0$ indicate a synergistic interaction of the two agents.

treatment with OTX015 and ibrutinib or venetoclax is synergistically lethal in all three RT-DLBCL cell-types $[27,36,49]$. The synergistic activity of OTX015 with venetoclax may be partially explained by OTX015mediated decline in the levels of MCL1 (vide infra Fig. 5E). 

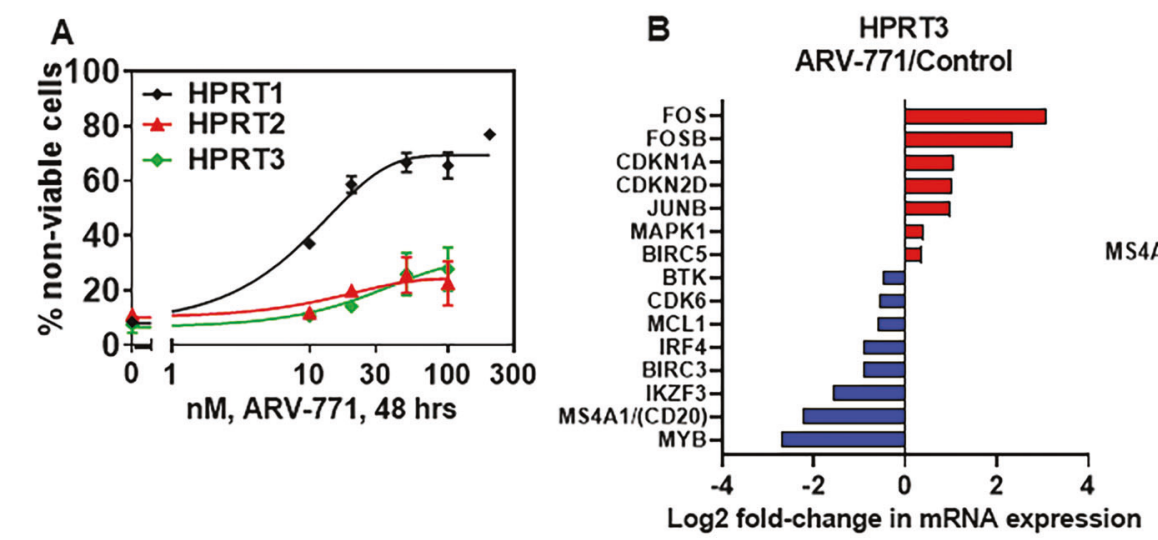

C HPRT2 HPRT2
$-771 /$ Control

Lold-change in mRNA expression
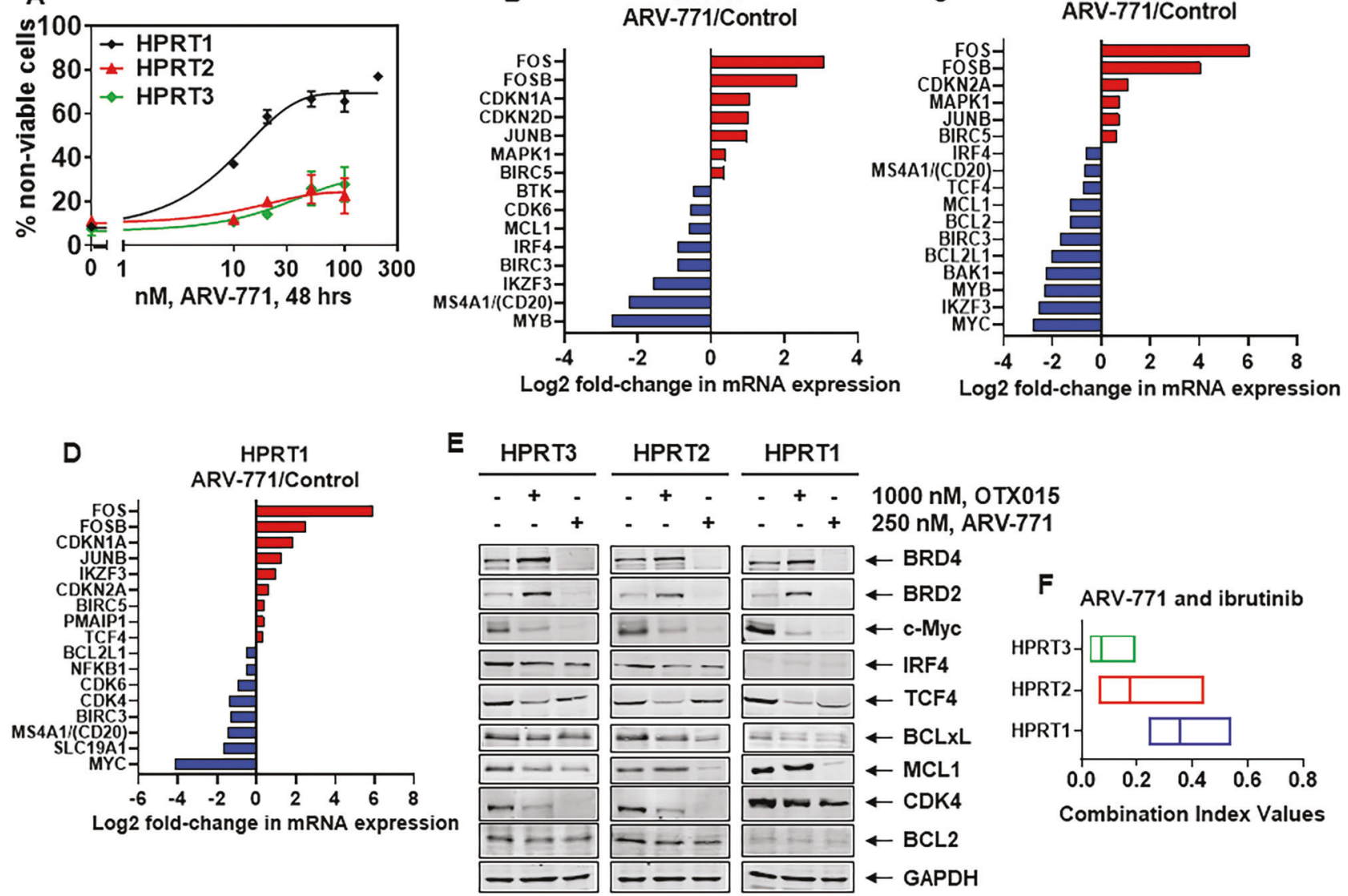

Fig. 5 Treatment with BET-PROTAC ARV-771 depletes c-Myc, CDK4, TCF4, IRF4, and Bcl-xL expression and exerts synergistic lethal activity with ibrutinib in RT-DLBCL cells. A RT-DLBCL cells were treated with the indicated concentrations of ARV-771 for $48 \mathrm{~h}$. At the end of treatment, the \% of To-Pro- 3 iodide-positive, nonviable cells was determined by flow cytometry. Mean of three experiments \pm SEM. B-D Comparison of selected, significantly altered $(\geq 1.25$ fold change up or down and $p$-value $<0.05$ relative to untreated control), mRNA expression changes detected by RNA-Seq analysis (on biologic triplicates) in HPRT3, HPRT2, and HPRT1 cells

\section{Preclinical efficacy of BET-PROTAC alone and in combination with ibrutinib or venetoclax against RT-DLBCL cells}

We had previously shown that BET-PROTACs degrade BET proteins and cause more profound perturbations in mRNA and protein levels, especially of c-Myc and other super-enhancer driven oncogenes, as well induce more apoptosis than BETi in NHL cells [43]. Therefore, we next evaluated effects of the BET-PROTAC ARV-771 on mRNA and protein expressions of RT-DLBCL-relevant oncogenes, as well as on survival of the RT-DLBCL celltypes. Figure 5A shows that ARV-771 induced greater lethality in HPRT1 $(\sim 65 \%$ loss of viability due to exposure to $50-100 \mathrm{nM}$ of ARV-771), compared to HPRT3 and HPRT2 cells, which exhibited $\sim 20 \%$ loss of viability following treatment with BET-PROTAC ARV-771 for $8 \mathrm{~h}$. E HPRT3, HPRT2, and HPRT1 cells were treated with $1000 \mathrm{nM}$ of OTX015 or $250 \mathrm{nM}$ of ARV-771 for $24 \mathrm{~h}$. Immunoblot analyses were conducted on the cell lysates. The expression levels GAPDH in the cell lysates served as the loading control. F RT-DLBCL cells were treated with ARV-771 (dose range: $20-250 \mathrm{nM}$ ) and/or ibrutinib (dose range: 2-10 $\mu \mathrm{M})$ for $48 \mathrm{~h}$. The $\%$ of non-viable cells was determined by flow cytometry. Combination index (CI) values were calculated with CompuSyn and boxplots were graphed with GraphPad V8. CI values $<1.0$ indicate a synergistic interaction of the two agents.

following exposure to $50-100 \mathrm{nM}$ of ARV-771. Concomitantly, RNA-Seq analyses demonstrated that ARV-771 induced more mRNA perturbations (up or downregulations) in HPRT1 compared to HPRT3 and HPRT2 cells (Fig. S8A-C). There were also more gene expression perturbations that overlapped between OTX015 and ARV-771 treatments in HPRT1 compared to HPRT3 and HPRT2 cells (Figs. S8D-F). RNA-Seq data showed $\log 2$-fold decline in the expression levels of MYB, IKZF3, IRF4, BIRC3, MCL1, CDK6, and BTK, with increases in CDKN1A and CDKN2D in HPRT3 and HPRT2 cells (Fig. 5B, C). Due to MYC amplification and increased expression of c-Myc, ARV-771 treatment caused more pronounced repression of MYC and its target SLC19A1 (folate transporter) in HPRT1 cells (Fig. 5D) [50]. As previously reported in other cell types, in the three RT-DLBCL cells also, treatment with 


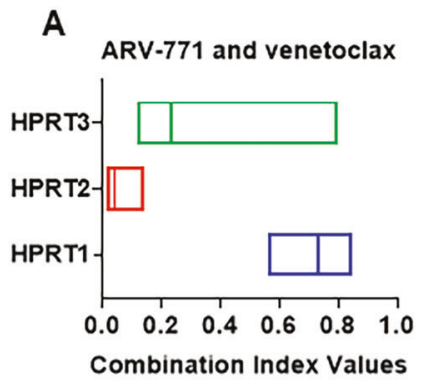

B

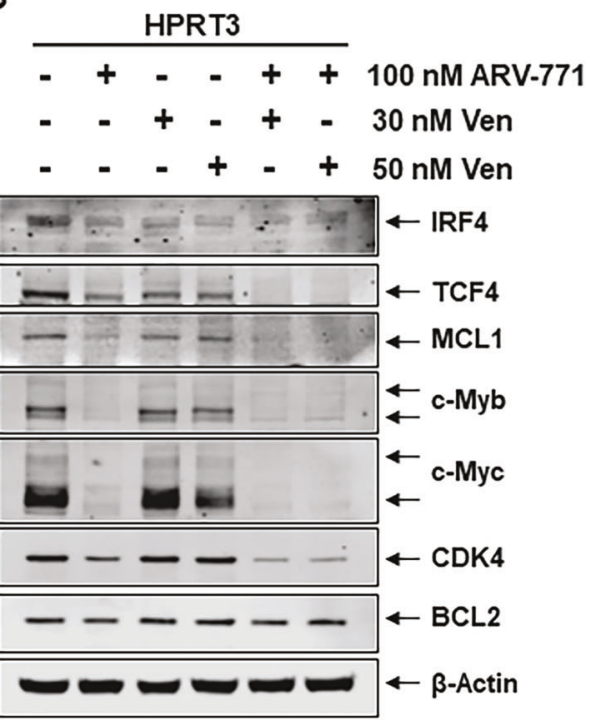

Fig. 6 Co-treatment with ARV-771 and venetoclax (ABT-199) reduces lymphoma burden in the spleen and liver as well as significantly improves survival of NSG mice engrafted with clonallyrelated HPRT3-DLBCL cells. A RT-DLBCL cells were treated with ARV-771 (dose range: $20-250 \mathrm{nM}$ ) and venetoclax (dose range: 20-100 nM) for $48 \mathrm{~h}$. The $\%$ of non-viable cells was determined by flow cytometry. Combination index $(\mathrm{CI})$ values were calculated with CompuSyn and boxplots were generated with GraphPad V8. CI values $<1.0$ indicate a synergistic interaction of the two agents. B HPRT3 cells were treated with ARV-771 and venetoclax (Ven) as indicated for $24 \mathrm{~h}$. At the end of treatment, total cell lysates were prepared and immunoblot analyses were conducted. The expression levels of $\beta$ Actin in the cell lysates served as the loading control. C, D. HPRT3 cells were engrafted into NSG mice by lateral tail-vein injection.

BETi OTX015 induced BRD4 levels (Fig. 5E) [43]. In contrast, ARV-771 treatment depleted BRD4 and BRD2 levels in the three RT-DLBCL cell-types (Fig. 5E). Although ARV-771 exerts similar effects on protein expressions of the genes shown in Fig. 5E, HPRT1 cells express higher levels of c-Myc, TCF4, MCL1, and CDK4 on which they may be more dependent for growth and survival. Therefore, more pronounced decline in the levels of these proteins due to ARV-771 treatment may exert greater lethality in HPRT1 compared to HPRT3 and HPRT2 cells (Fig. 5E). Similar to BETi-based combinations, we
D

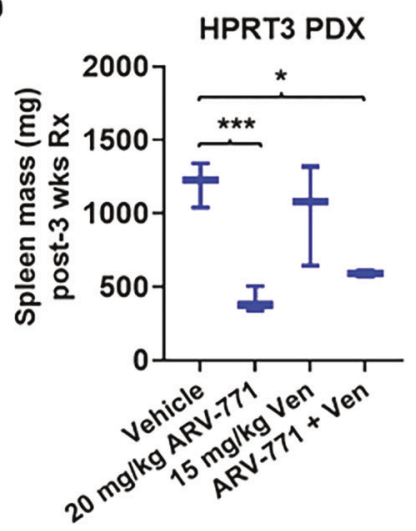

E

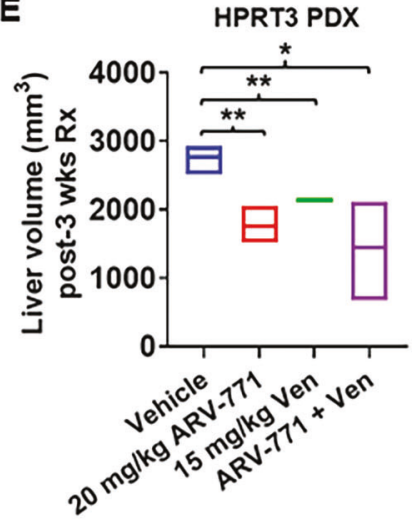

Following documentation of engraftment, mice were treated with ARV-771 and venetoclax (Ven), as indicated for 3 weeks. Mice were sacrificed and spleen length and spleen mass were determined. ${ }^{*} p<$ $0.05 ; * * * p<0.005$ as determined by two-tailed, unpaired $t$-test. E HPRT3 cells were engrafted into NSG mice by lateral tail-vein injection. Following documentation of engraftment, mice were treated with ARV-771 and venetoclax (Ven) as indicated for 3 weeks. Mice were imaged by MRI to document effects of treatment on liver volume. ${ }^{*} p<0.05 ;{ }^{*} p<0.01$ as determined by two-tailed, unpaired $t$ test. F Kaplan-Meier survival plot of NSG mice treated with $20 \mathrm{mg} / \mathrm{kg}$ of ARV-771 and/or $15 \mathrm{mg} / \mathrm{kg}$ venetoclax for 3 weeks. Significant differences in overall survival between the vehicle and treated mice were determined by Mantel-Cox log rank test.

also determined lethal effects of co-treatment with ARV771 and ibrutinib or venetoclax in the three RT-DLBCL cell types. Co-treatment with ARV-771 and ibrutinib or venetoclax exerted synergistic lethality in all three RT-DLBCL cell-types (Figs. 5F and 6A). This synergy with venetoclax may be partially explained by ARV-771-mediated decline in the levels of MCL1. Finally, we determined the in vivo efficacy of BET-PROTAC ARV-771 and/or venetoclax against the PDX model of HPRT3 cells administered via tail-vein infusion and engrafted in NSG mice. Ex vivo exposure of HPRT3 cells to ARV-771 and venetoclax 
mediated more decline in IRF4, TCF4, c-Myc, c-Myb, MCL1, and CDK4 protein levels, as compared to treatment with ARV-771 alone (Fig. 6B). Following engraftment and 7-days post-implantation, compared to vehicle control, daily treatment for three weeks with ARV-771 and venetoclax significantly reduced the spleen length and mass, as well as reduced the liver volume, highlighting reduction in tumor volume in the mice engrafted with HPRT3 cells (Fig. 6CE). Notably, mice co-treated with ARV-771 and venetoclax demonstrated significantly improved overall survival, as compared to mice treated with vehicle control, or with venetoclax or ARV-771 alone $(p<0.05)$ (Fig. $6 \mathrm{~F})$.

\section{Discussion}

To address the preceding absence and unmet need for preclinical RT-DLBCL models, we report here generation and characterization of the first-ever, PDX models of CLR and CLUR RT-DLBCLs. Utilizing traditional cytogenetics, array-CGH, NGS, ATAC-Seq, ChIP-Seq, scRNA-Seq, and Western analyses, a full characterization of genomics/epigenomics and gene-expression profiles of the RT-DLBCL models is also presented. The large array of genetic alterations in HPRT3 and HPRT2 cells, representative of CLR or CLUR RT-DLBCLs, is a reflection of the rich profile of recurrent mutations previously described in the coding and non-coding genes during CLL progression $[51,52]$. The high numbers of genetic alterations in the CLR and CLUR RT-DLBCLs complicated and limited the identification of genomic targets for therapy. Therefore, it was necessary to correlate the gene expressions in the RTDLBCL cells with sensitivity to standard and novel therapeutic agents. These correlations demonstrated that, although individually the drugs are only modestly active, combinations of BETi or BET-PROTAC with ibrutinib or venetoclax were synergistically lethal against the CLR and CLUR RT-DLBCL cells. Notably, co-treatment with BETPROTAC and venetoclax also reduced lymphoma burden and improved survival of NSG mice engrafted with CLR RT-DLBCL cells.

Based on the presence of IGH gene rearrangement, HPRT3 cells were CLR, HPRT2 CLUR, and HPRT1 were of indeterminate clonal origin, compared to their respective preceding CLL clones. Phenotypically, all three cells expressed CD20, but only HPRT3 and HPRT2 cells expressed high levels of IRF4, suggesting their postgerminal cell of origin [2, 5]. These observations were consistent with immunohistochemistry-determined protein expressions documented in the large cohort of 52 RTDLBCLs. They showed uniformly positive expression of PAX5 and IRF4 (MUM1), whereas CD10 expression was absent. Phenotypic protein expression patterns indicated that HPRT3 and HPRT2 cells were of ABC sub-type of DLBCL, as were the vast majority of RT-DLBCLs [2, 5]. Although HPRT2 and HPRT1 cells were low-expressers of CD5 and CD23, HPRT3 cells expressed higher surface CD5 and $\mathrm{CD} 23$, as was also seen in some samples among the larger cohort of RT-DLBCLs. HPRT1 cells expressed high levels of CD10 and BCL6, which was consistent with their GCB cell phenotype [2]. These cells were also low expressers of PD-1, which is rarely expressed by de novo DLBCL [2, 5]. Additionally, compared to HPRT3 and HPRT2 cells, HPRT1 cells expressed higher levels of cMyc and Ki67, which is consistent with high cell cycle Sphase status, and is explained by the presence of MYC amplification in HPRT1 cells. Consistent with other reports, the RT-DLBCLs presented here were also negative for EB virus infection $[2,5]$. Also, similar to prior reports, cytogenetics analysis showed profound aneuploidy with presence of marked chromosomal tetrasomy and trisomy in CLR HPRT3 cells [8], compared to CLUR HPRT2 and HPRT1 cells. This was corroborated via array-CGH and low-pass WGS, which demonstrated large numbers of chromosomal amplifications and losses as well as arrays of copy gains and losses, respectively, in the CLR HPRT3 compared to either CLUR HPRT2 or the de novo-GCBDLBCL HPRT1 cells. Whole exome NGS analysis of 300 genes also underscored the high numbers of genetic mutations in HPRT3 and HPRT2 cells compared to the mutationally relatively more quiescent HPRT1 cells. Overall, observed mutations in the RT-DLBCL cells involved genes of epigenetic modifiers, transcription factors, surface receptors, signaling kinases, DNA damage/response pathway, and apoptosis threshold regulators. Although genetic alterations in NOTCH, MYC, and DNA damage/repair pathways suggested likely sensitivity of HPRT1 cells to agents that target these dependencies, presence of multiple genetic alterations in HPRT3 and HPRT2 cells defied clear delineation of dependencies just based on their profile of genetic mutations. However, epigenetic and geneexpression analyses illuminated potentially more targetable dependencies in HPRT3 and HPRT2 cells.

All three RT-DLBCL cells displayed more open chromatin and increased H3K27Ac occupancy at the SEs/Es, as compared to normal CD34+ progenitor cells. More granularly, increased chromatin accessibility at the MYC, TCF4, and PLC $\gamma 2$ loci was also accompanied by higher H3K27Ac occupancy at the Es of these genes. Additionally, open chromatin at the IRF4 and BCL2 loci was associated with increased H3K27Ac occupancy at their Es, but only in HPRT3 and HPRT2, not in HPRT1 cells. Notably, high copy-gains of TCF4 in HPRT3 and HPRT2 cells, observed via low-pass WGS, was associated with increased chromatin accessibility and activity of TCF4 SE, highlighted by increased occupancy with H3K27Ac and BRD4, as recently 
reported [28]. This report had also shown that copy gains of TCF4 at 18q21.1 are commonly found in ABC subtype of de novo DLBCL, which explains why TCF4 copy gains were present in the ABC subtype of HPRT3 and HPRT2 RT-DLBCL cells [28]. BRD4 occupancy was also increased at the active enhancers of IRF4, PAX5, and MYC in HPRT3 and HPRT2 cells. Consistent with its highly aneuploid status and highest level of active chromatin, scRNASeq analyses showed that HPRT3 cells exhibited highest number of transcriptionally active cell-clusters, although high mRNA expressions of TCF4, PAX5, and IRF4 were observed in HPRT3 and HPRT2 cells. Even though IRF4 and TCF4 induce MYC, scRNA-Seq showed only a muted MYC mRNA expression in HPRT3 and HPRT2 cells $[28,48]$. Conversely, uniformly high Myc expression in HPRT1 cells was explained by amplification of MYC and high occupancy of MYC chromatin by H3K27Ac and BRD4. Although not shown here, high TCF4 expression in HPRT1 cells may be due to high H3K27Ac and BRD4 occupancy at the SE of TCF4, and by MYC-facilitated transcription of TCF4 [28, 53]. In all the RT-DLBCL cells, their mRNA levels, determined by scRNA-Seq, also generally corresponded with the protein levels of BCL2 family of proteins, including BCL2, MCL1, Bcl-xL, BAX, BAK, BIM (BCL2L11), as well as with the protein expression of c-IAP2 (BIRC3), XIAP, and Ki67 (MKI67).

Overall, the mRNA and protein expression levels correlated with specific drug-sensitivities of the RT-DLBCL cells. Notably, high BCL2 and Bcl-xL, concomitantly with low expression of MCL1 in HPRT3 and HPRT2 versus HPRT1 cells, respectively correlated with higher sensitivity to venetoclax and A-1155463 (Bcl-xL inhibitor) and lesser sensitivity to AZD-5991 (MCL1 inhibitor)-induced cell lethality. Additionally, in HPRT3 and HPRT2 versus HPRT1 cells, high NFkB2/p52 levels conferred relative resistance to ibrutinib [37]. Although of GCB-DLBCL subtype, HPRT1 cells exhibited more sensitivity to ibrutinib, likely due to higher activities and levels of p-BTK and pPLC $\gamma 2$ (Fig. 4F). Higher cereblon and IKZF1/3 levels in HPRT1 cells, compared to HPRT3 and HPRT2 cells (Fig. 5C), may also explain the greater sensitivity of HPRT1 cells to lenalidomide, which induces cereblonmediated degradation of IKZF1/3 in transformed B cells $[41,42]$. Conversely, considerably higher levels of sensitivity of HPRT1 compared to HPRT3 and HPRT2 cells to BETis OTX015 and ABBV-075, and to the BET-PROTAC ARV-771, correlated with higher protein expressions of BRD4, c-Myc, and TCF4 in HPRT1 cells. The latter geneexpressions are well known to be driven by super-enhancers with high BRD4 occupancy [19, 25, 28]. It is noteworthy that previously reported mechanisms of resistance to BETi, such as high levels/activity of DUB3, TRIM33, SPOP or pAKT, were not observed and did not appear to contribute to the disparate sensitivity of HPRT3 and HPRT2 versus HPRT1 cells to BETi [44-46]. However, markedly higher expression levels of IRF4 did mechanistically contribute to partial resistance of HPRT3 and HPRT2 cells to BETi treatment, since IRF4 knockout via CRISPR-Cas9 partially re-sensitized the cells to BETi. IRF4 depletion resulted in reduced nuclear c-Myc expression, which would explain increased sensitivity of HPRT3 and HPRT2 cells to BETiinduced lethality. To determine whether the modest singleagent activity of BETi, or ARV-771 could be improved against the RT-DLBCL cells, especially against the poorrisk CLR-RT-DLBCL HPRT3 cells, we interrogated the lethal activity of BETi or ARV-771 combined with venetoclax or ibrutinib. Indeed, co-treatment with BETi or ARV771 exerted synergistic in vitro lethality against all three DLBCLs. Consistent with higher in vitro activity of venetoclax against HPRT3 cells, co-treatment with relatively low doses of ARV-771 and venetoclax significantly reduced in vivo HPRT3 cell burden and improved survival of immune-depleted mice engrafted with HPRT3 cells, without resulting in appreciable host toxicity. These findings strongly support further in vivo testing and development of BETi or BET-PROTAC-based combinations with other BH3 mimetics, BCR-signaling kinase inhibitors, as well as with other novel targeted agents, utilizing the newly established and characterized models of RT-DLBCLs.

Acknowledgements The authors would like to thank the Sequencing and Microarray Core Facility, Flow Cytometry and Cellular Imaging (FCCI) Core Facility, which are supported by the MD Anderson Cancer Center Support Grant 5 P30 CA016672-40. C.C. acknowledges support from CPRIT RP170005, NIEHS CG-CPEH P30 ES030285 and the NCI Cancer Center Support Grant P30 CA125123 to the Dan L. Duncan Cancer Center. C.M.C. acknowledges support from the National Institutes of Health (grant number R35 CA197589) and Arvinas. This research is supported in part by the MD Anderson Cancer Center Leukemia SPORE (P50 CA100632).

Author contributions K.N.B. designed the study. D.P. and C.C. performed bioinformatics analyses. W.F., C.P.M., C.B., Q.D., H.Y., B.L., D.T.S., W.J., M.R.G. and J.D.K. performed research and analyzed the data. N.J., J.B., A.F. and C.M.C. contributed critical reagents. K.N.B. and W.F. wrote the paper.

\section{Compliance with ethical standards}

Conflict of interest C.M.C. is the founder and Chief Scientific Advisor of, and possesses an equity ownership stake in, Arvinas, Inc. All other authors state that they have no conflict of interest to declare.

Publisher's note Springer Nature remains neutral with regard to jurisdictional claims in published maps and institutional affiliations.

Open Access This article is licensed under a Creative Commons Attribution 4.0 International License, which permits use, sharing, adaptation, distribution and reproduction in any medium or format, as long as you give appropriate credit to the original author(s) and the source, provide a link to the Creative Commons license, and indicate if 
changes were made. The images or other third party material in this article are included in the article's Creative Commons license, unless indicated otherwise in a credit line to the material. If material is not included in the article's Creative Commons license and your intended use is not permitted by statutory regulation or exceeds the permitted use, you will need to obtain permission directly from the copyright holder. To view a copy of this license, visit http://creativecommons. org/licenses/by/4.0/.

\section{References}

1. Jamroziak K, Tadmor T, Robak T, Polliack A. Richter syndrome in chronic lymphocytic leukemia: updates on biology, clinical features and therapy. Leuk Lymphoma. 2015;56:1949-58.

2. Rossi D, Spina V, Gaidano G. Biology and treatment of Richter syndrome. Blood. 2018;131:2761-72.

3. Chigrinova E, Rinaldi A, Kwee I, Rossi D, Rancoita PM, Strefford JC, et al. Two main genetic pathways lead to the transformation of chronic lymphocytic leukemia to Richter syndrome. Blood. 2013;122:2673-82.

4. Kadri S, Lee J, Fitzpatrick C, Galanina N, Sukhanova M, Venkataraman $\mathrm{G}$, et al. Clonal evolution underlying leukemia progression and Richter transformation in patients with ibrutinibrelapsed CLL. Blood Adv. 2017;1:715-27.

5. Rossi D, Gaidano G. Richter syndrome: pathogenesis and management. Semin Oncol. 2016;43:311-9.

6. Wang Y, Tschautscher MA, Rabe KG, Call TG, Leis JF, Kenderian SS, et al. Clinical characteristics and outcomes of Richter transformation: experience of 204 patients from a single center. Haematologica. 2020;105:765-73.

7. Fabbri G, Khiabanian H, Holmes AB, Wang J, Messina M, Mullighan CG, et al. Genetic lesions associated with chronic lymphocytic leukemia transformation to Richter syndrome. J Exp Med. 2013;210:2273-88.

8. Miller CR, Ruppert AS, Heerema NA, Maddocks KJ, Labanowska $\mathrm{J}$, Breidenbach $\mathrm{H}$, et al. Near-tetraploidy is associated with Richter transformation in chronic lymphocytic leukemia patients receiving ibrutinib. Blood Adv. 2017;1:1584-8.

9. Woroniecka R, Rymkiewicz G, Grygalewicz B, Blachnio K, Rygier J, Jarmuz-Szymczak M, et al. Cytogenetic and flow cytometry evaluation of Richter syndrome reveals MYC, CDKN2A, IGH alterations with loss of CD52, CD62L and increase of CD71 antigen expression as the most frequent recurrent abnormalities. Am J Clin Pathol. 2015;143:25-35.

10. Behdad A, Griffin B, Chen YH, Ma S, Kelemen K, Lu X, et al. PD-1 is highly expressed by neoplastic B-cells in Richter transformation. Br J Haematol. 2019;185:370-3.

11. Davids MS, Roberts AW, Seymour JF, Pagel JM, Kahl BS, Wierda WG, et al. Phase I first-in-human study of venetoclax in patients with relapsed or refractory non-Hodgkin lymphoma. J Clin Oncol. 2017;35:826-33.

12. Ding W. Richter transformation in the era of novel agents. Hematol Am Soc Hematol Educ Program. 2018;2018:256-63.

13. Ding W, LaPlant BR, Call TG, Parikh SA, Leis JF, He R, et al. Pembrolizumab in patients with CLL and Richter transformation or with relapsed CLL. Blood. 2017;129:3419-27.

14. Eyre TA, Schuh A. An update for Richter syndrome - new directions and developments. Br J Haematol. 2017;178:508-20.

15. Langerbeins P, Busch R, Anheier N, Durig J, Bergmann M, Goebeler ME, et al. Poor efficacy and tolerability of R-CHOP in relapsed/refractory chronic lymphocytic leukemia and Richter transformation. Am J Hematol. 2014;89:E239-43.

16. Chapuy B, Stewart C, Dunford AJ, Kim J, Kamburov A, Redd RA, et al. Molecular subtypes of diffuse large B cell lymphoma are associated with distinct pathogenic mechanisms and outcomes. Nat Med. 2018;24:679-90.

17. Pasqualucci L, Dalla-Favera R. Genetics of diffuse large B-cell lymphoma. Blood. 2018;131:2307-19.

18. Schmitz R, Wright GW, Huang DW, Johnson CA, Phelan JD, Wang JQ, et al. Genetics and pathogenesis of diffuse large B-cell lymphoma. N. Engl J Med. 2018;378:1396-407.

19. Bradner JE, Hnisz D, Young RA. Transcriptional addiction in cancer. Cell. 2017;168:629-43.

20. Shi J, Vakoc CR. The mechanisms behind the therapeutic activity of BET bromodomain inhibition. Mol Cell. 2014;54:728-36.

21. Stathis A, Bertoni F. BET proteins as targets for anticancer treatment. Cancer Disco. 2018;8:24-36.

22. Itzen F, Greifenberg AK, Bosken CA, Geyer M. Brd4 activates P$\mathrm{TEFb}$ for RNA polymerase II CTD phosphorylation. Nucleic Acids Res. 2014;42:7577-90.

23. Winter GE, Mayer A, Buckley DL, Erb MA, Roderick JE, Vittori $\mathrm{S}$, et al. BET bromodomain proteins function as master transcription elongation factors independent of CDK9 recruitment. Mol Cell. 2017;67:5-18.e9.

24. Jia Y, Chng WJ, Zhou J. Super-enhancers: critical roles and therapeutic targets in hematologic malignancies. J Hematol Oncol. 2019;12:77.

25. Loven J, Hoke HA, Lin CY, Lau A, Orlando DA, Vakoc CR, et al. Selective inhibition of tumor oncogenes by disruption of superenhancers. Cell. 2013;153:320-34.

26. Zou Z, Huang B, Wu X, Zhang H, Qi J, Bradner J, et al. Brd4 maintains constitutively active NF-kappaB in cancer cells by binding to acetylated RelA. Oncogene. 2014;33:2395-404.

27. Sun B, Shah B, Fiskus W, Qi J, Rajapakshe K, Coarfa C, et al. Synergistic activity of BET protein antagonist-based combinations in mantle cell lymphoma cells sensitive or resistant to ibrutinib. Blood. 2015;126:1565-74.

28. Jain N, Hartert K, Tadros S, Fiskus W, Havranek O, Ma MCJ, et al. Targetable genetic alterations of TCF4 (E2-2) drive immunoglobulin expression in diffuse large B cell lymphoma. Sci Transl Med. 2019;11:eaav5599.

29. Vaisitti T, Braggio E, Allan JN, Arruga F, Serra S, Zamo A, et al. Novel Richter syndrome xenograft models to study genetic architecture, biology, and therapy responses. Cancer Res. 2018;78:3413-20.

30. Mill CP, Fiskus W, DiNardo CD, Qian Y, Raina K, Rajapakshe K, et al. RUNX1-targeted therapy for AML expressing somatic or germline mutation in RUNX1. Blood. 2019;134:59-73.

31. Saenz DT, Fiskus W, Mill CP, Perera D, Manshouri T, Lara BH, et al. Mechanistic basis and efficacy of targeting the beta-cateninTCF7L2-JMJD6-c-Myc axis to overcome resistance to BET inhibitors. Blood. 2020;135:1255-69.

32. Chapuy B, McKeown MR, Lin CY, Monti S, Roemer MG, Qi J, et al. Discovery and characterization of super-enhancer-associated dependencies in diffuse large B cell lymphoma. Cancer Cell. 2013;24:777-90.

33. Pastore A, Gaiti F, Lu SX, Brand RM, Kulm S, Chaligne R, et al. Corrupted coordination of epigenetic modifications leads to diverging chromatin states and transcriptional heterogeneity in CLL. Nat Commun. 2019;10:1874.

34. Ott CJ, Federation AJ, Schwartz LS, Kasar S, Klitgaard JL, Lenci $\mathrm{R}$, et al. Enhancer architecture and essential core regulatory circuitry of chronic lymphocytic leukemia. Cancer Cell. 2018;34:982-95 e7.

35. Roberts AW, Davids MS, Pagel JM, Kahl BS, Puvvada SD, Gerecitano JF, et al. Targeting BCL2 with venetoclax in relapsed chronic lymphocytic leukemia. N. Engl J Med. 2016;374:311-22.

36. Fiskus W, Cai T, DiNardo CD, Kornblau SM, Borthakur G, Kadia $\mathrm{TM}$, et al. Superior efficacy of cotreatment with BET protein 
inhibitor and BCL2 or MCL1 inhibitor against AML blast progenitor cells. Blood. Cancer J. 2019;9:4.

37. Rahal R, Frick M, Romero R, Korn JM, Kridel R, Chan FC, et al. Pharmacological and genomic profiling identifies NF-kappaBtargeted treatment strategies for mantle cell lymphoma. Nat Med. 2014;20:87-92.

38. Chiron D, Di Liberto M, Martin P, Huang X, Sharman J, Blecua $\mathrm{P}$, et al. Cell-cycle reprogramming for PI3K inhibition overrides a relapse-specific C481S BTK mutation revealed by longitudinal functional genomics in mantle cell lymphoma. Cancer Disco. 2014;4:1022-35.

39. Wiestner A. Emerging role of kinase-targeted strategies in chronic lymphocytic leukemia. Blood. 2012;120:4684-91.

40. Woyach JA, Johnson AJ. Targeted therapies in CLL: mechanisms of resistance and strategies for management. Blood. 2015;126:471-7.

41. Zhu YX, Braggio E, Shi CX, Bruins LA, Schmidt JE, Van Wier S, et al. Cereblon expression is required for the antimyeloma activity of lenalidomide and pomalidomide. Blood. 2011;118:4771-9.

42. Petzold G, Fischer ES, Thoma NH. Structural basis of lenalidomide-induced CK1alpha degradation by the CRL4 (CRBN) ubiquitin ligase. Nature. 2016;532:127-30.

43. Sun B, Fiskus W, Qian Y, Rajapakshe K, Raina K, Coleman KG, et al. BET protein proteolysis targeting chimera (PROTAC) exerts potent lethal activity against mantle cell lymphoma cells. Leukemia. 2018;32:343-52.

44. Dai X, Gan W, Li X, Wang S, Zhang W, Huang L, et al. Prostate cancer-associated SPOP mutations confer resistance to BET inhibitors through stabilization of BRD4. Nat Med. 2017;23:1063-71.
45. Jin $\mathrm{X}$, Yan $\mathrm{Y}$, Wang D, Ding D, Ma T, Ye Z, et al. DUB3 promotes BET inhibitor resistance and cancer progression by deubiquitinating BRD4. Mol Cell. 2018;71:592-605 e4.

46. Shi X, Mihaylova VT, Kuruvilla L, Chen F, Viviano S, Baldassarre M, et al. Loss of TRIM33 causes resistance to BET bromodomain inhibitors through MYC- and TGF-beta-dependent mechanisms. Proc Natl Acad Sci USA. 2016;113:E4558-66.

47. Devaraj SG, Fiskus W, Shah B, Qi J, Sun B, Iyer SP, et al. HEXIM1 induction is mechanistically involved in mediating antiAML activity of BET protein bromodomain antagonist. Leukemia. 2016;30:504-8.

48. Agnarelli A, Chevassut T, Mancini EJ. IRF4 in multiple myeloma-biology, disease and therapeutic target. Leuk Res. 2018;72:52-8.

49. Bui MH, Lin X, Albert DH, Li L, Lam LT, Faivre EJ, et al. Preclinical characterization of BET family bromodomain inhibitor ABBV-075 suggests combination therapeutic strategies. Cancer Res. 2017;77:2976-89.

50. Vazquez A, Tedeschi PM, Bertino JR. Overexpression of the mitochondrial folate and glycine-serine pathway: a new determinant of methotrexate selectivity in tumors. Cancer Res. 2013;73:478-82.

51. Landau DA, Tausch E, Taylor-Weiner AN, Stewart C, Reiter JG, Bahlo J, et al. Mutations driving CLL and their evolution in progression and relapse. Nature. 2015;526:525-30.

52. Puente XS, Bea S, Valdes-Mas R, Villamor N, Gutierrez-Abril J, Martin-Subero JI, et al. Non-coding recurrent mutations in chronic lymphocytic leukaemia. Nature. 2015;526:519-24.

53. Lin CY, Loven J, Rahl PB, Paranal RM, Burge CB, Bradner JE, et al. Transcriptional amplification in tumor cells with elevated cMyc. Cell. 2012;151:56-67. 